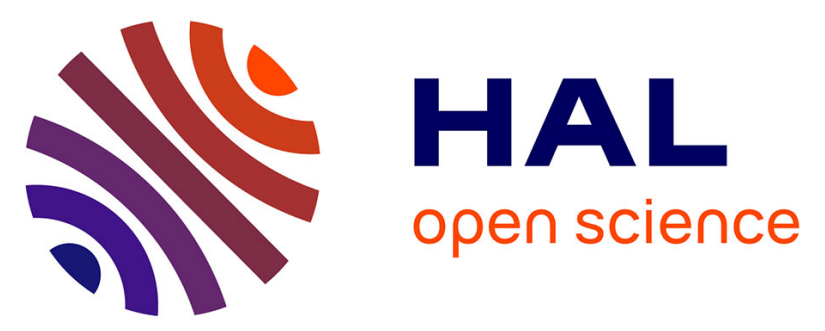

\title{
Plasma amyloid $\beta$ 40/42 ratio predicts cerebral amyloidosis in cognitively normal individuals at risk for Alzheimer's disease
}

Andrea Vergallo, Lucile Mégret, Simone Lista, Enrica Cavedo, Henrik Zetterberg, Kaj Blennow, Eugeen Vanmechelen, Ann de Vos, Marie-Odile Habert, Marie-Claude Potier, et al.

\section{To cite this version:}

Andrea Vergallo, Lucile Mégret, Simone Lista, Enrica Cavedo, Henrik Zetterberg, et al.. Plasma amyloid $\beta$ 40/42 ratio predicts cerebral amyloidosis in cognitively normal individuals at risk for Alzheimer's disease. Alzheimer's \& Dementia: the Journal of the Alzheimer's Association, 2019, 15 (6), pp.764 775. 10.1016/j.jalz.2019.03.009 . hal-03484725

\author{
HAL Id: hal-03484725 \\ https://hal.science/hal-03484725
}

Submitted on 20 Dec 2021

HAL is a multi-disciplinary open access archive for the deposit and dissemination of scientific research documents, whether they are published or not. The documents may come from teaching and research institutions in France or abroad, or from public or private research centers.
L'archive ouverte pluridisciplinaire HAL, est destinée au dépôt et à la diffusion de documents scientifiques de niveau recherche, publiés ou non, émanant des établissements d'enseignement et de recherche français ou étrangers, des laboratoires publics ou privés.

\section{(ㄷ)(1) $\$$}

Distributed under a Creative Commons Attribution - NonCommerciall 4.0 International 
Version of Record: https://www.sciencedirect.com/science/article/pii/S1552526019300822

Aeantreccifintes 4 e098eb478b15286285c7e6a5e 78f5f3

\section{Plasma amyloid $\beta$ 40/42 ratio predicts cerebral amyloidosis in cognitively normal individuals at risk for Alzheimer's disease}

Andrea Vergallo $\mathrm{a}^{\mathrm{a}, \mathrm{b}, \mathrm{c}, \boldsymbol{A} \rightarrow *}$

andrea.vergallo@icm-institute.org

Lucile Mégret $t^{d-1}$

Simone Lista ${ }^{a, b, ~ o, d ~}$

Enrica Cavedoa, b, o, d

Henrik Zetterberg, in i, f,g,h

Kaj Blennow

Eugeen Vanmechelen

Ann De Vosi

Marie-Odile Habert

Marie-Claude Potierm $m$

Bruno Dubois

Christian Neried

Harald Hampel $\left.\right|^{2-b-6-e r}$

and the INSIGHT-preAD study group

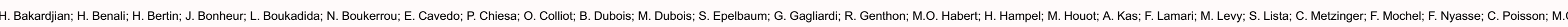
Potier; M. Revillon; A. Santos; K.S. Andrade; M. Sole; M. Surtee; Thiebaud de Schotten M; A. Vergallo; N. Youns

for the Alzheimer Precision Medicine Initiative (APMI)

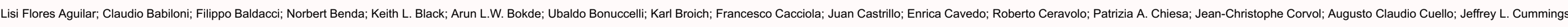

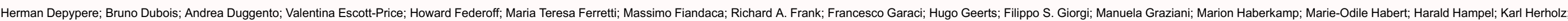

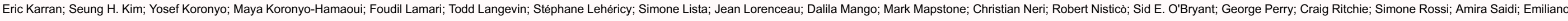
Santarnecchi; Lon S. Schneider; Olaf Sporns; Nicola Toschi; Steven R. Verdooner; Andrea Vergallo; Nicolas Villain; Lindsay A. Welikovitch; Janet Woodcock; Erfan Younesi

AXA Research Fund \& Sorbonne University Chair, Paris, France

Đas-Sorbonne University, GRC no 21, Alzheimer Precision Medicine (APM), AP-HP, Pitié-Salpêtrière Hospital, Boulevard de l'hôpital, Paris, France

르BBrain \& Spine Institute (ICM), INSERM U 1127, CNRS UMR 7225, Boulevard de l'hôpital, Paris, France

dcInstitute of Memory and Alzheimer's Disease (IM2A), Department of Neurology, Pitié-Salpêtrière Hospital, AP-HP, Boulevard de l'hôpital, Paris, France

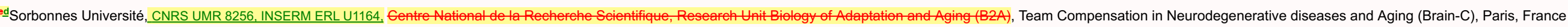

feInstitute of Neuroscience and Physiology, Department of Psychiatry and Neurochemistry, The Sahlgrenska Academy at the University of Gothenburg, Mölndal, Sweden

(C) 2019 published by Elsevier. This manuscript is made available under the CC BY NC user license

https://creativecommons.org/licenses/by-nc/4.0/ 


\section{${ }^{9 t}$ Clinical Neurochemistry Laboratory, Sahlgrenska University Hospital, MöIndal, Sweden}

hag Department of Molecular Neuroscience, UCL Institute of Neurology, London, UK

ihUK Dementia Research Institute, London, UK

i:ADx NeuroSciences NV, Ghent, Belgium

kisorbonne Université, CNRS, INSERM, Laboratoire d'Imagerie Biomédicale, Paris, France

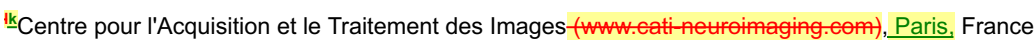

m!AP-HP, Hôpital Pitié-Salpêtrière, Département de Médecine Nucléaire, Paris, France

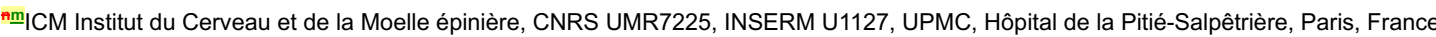

*Corresponding author. Tel.: +3314216 19 93; Fax:+331572740 27-sos.

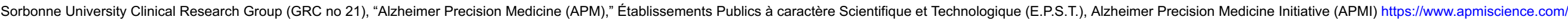

\section{Abstract}

Introduction

Blood-based biomarkers of pathophysiological brain amyloid $\beta(A \beta)$ accumulation, particularly for preclinical target and large-scale interventions, are warranted to effectively treatenrich Alzheimer's disease clinical trials and management.

\section{Methods}

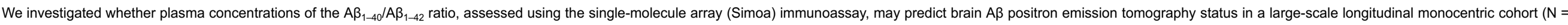

276) of older individuals with subjective memory complaints. We performed a hypothesis-driven investigation followed by a nonparametric, no-a-priori hypothesis study using machine learning

Results

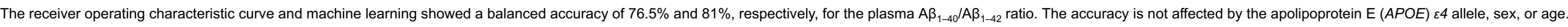
Discussion

Our results encourage an independent validation cohort study to validateconfirm the indication that the plasma $A \beta_{1-40} / A \beta_{1-42}$ ratio, assessed via Simoa, may improve future standard of care and clinical trial design

Keywords: Alzheimer's disease; Plasma amyloid $\beta$; Simoa immunoassay; Machine learning; Subjective memory complainers; Amyloid PET; Classification and regression trees (CART)

\section{Introduction}

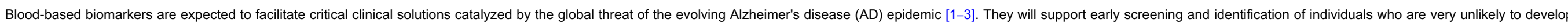

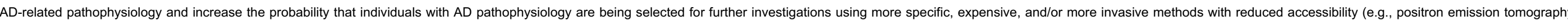

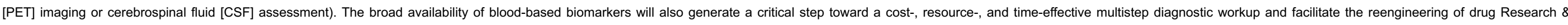
Development programspipelines, from proof of pharmacology to clinical trial design [4-8].

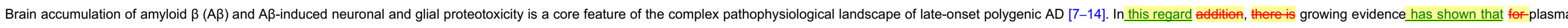

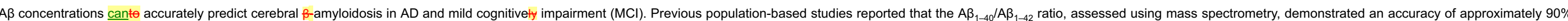

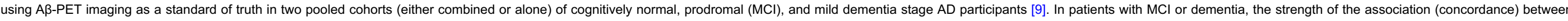

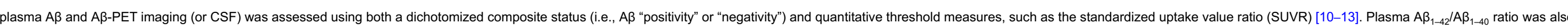




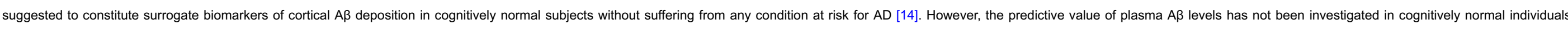

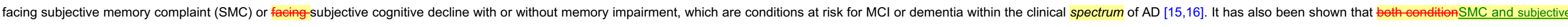

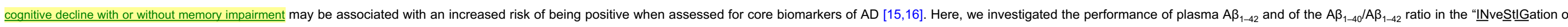

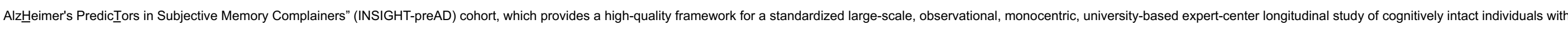

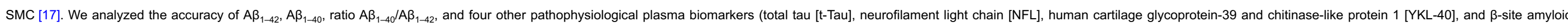

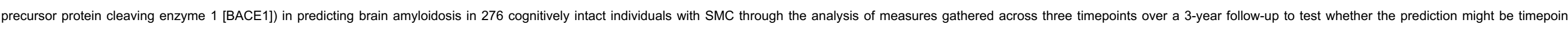

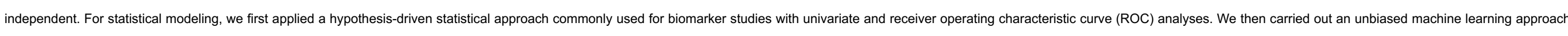

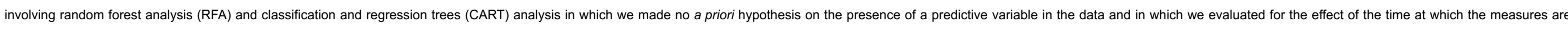

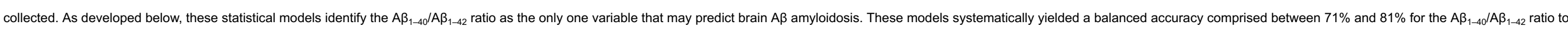

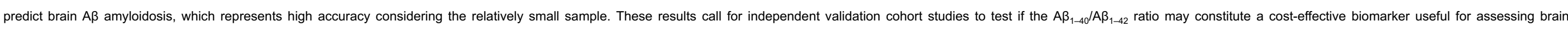
amyloidosis in individuals who are at risk for $A D$.

\section{Materials and methods}

\subsection{Study participants}

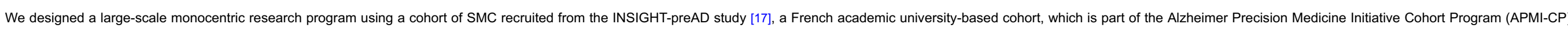

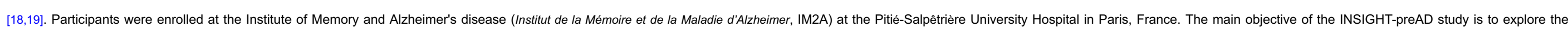
earliest preclinical stages of $\mathrm{AD}$ through intermediate to later stages until conversion to first cognitive symptoms, using clinical parameters and biomarkers associated with cognitive progression.

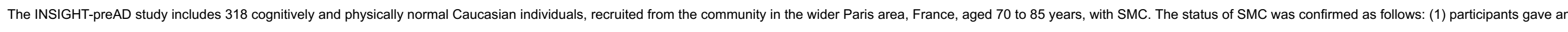

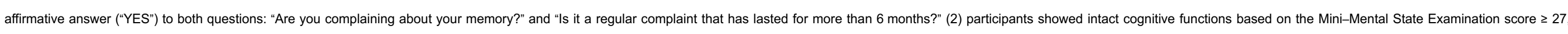

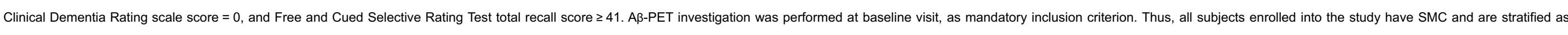

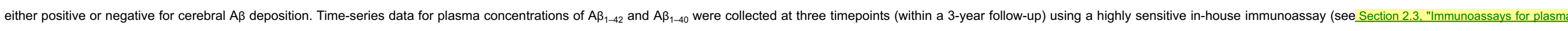

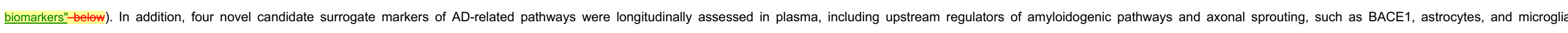

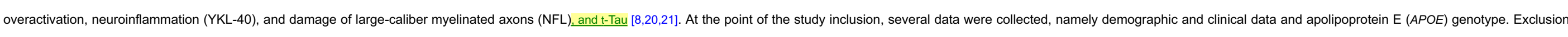

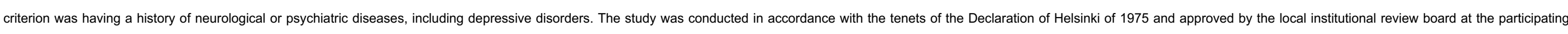
center. All participants or their representatives gave written informed consent for use of their clinical data for research purposes.

\subsection{Blood sampling and collection tube storage}

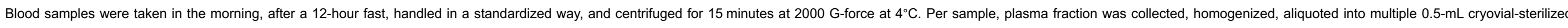
tubes, and finally stored at $-80^{\circ} \mathrm{C}$ within 2 hours from collection.

\subsection{Immunoassays for plasma biomarkers}

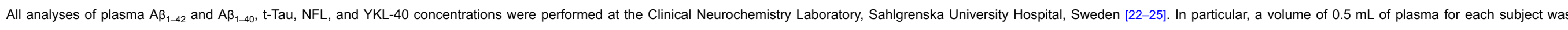
required for performing the analyses using the aforementioned platforms.

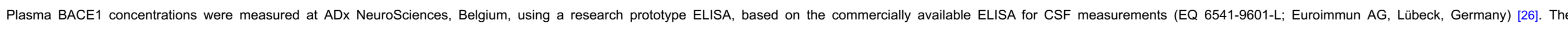
measurements of each biomarker were performed in one round of experiments, using the same batch of reagents, by board-certified laboratory technicians who were blinded to the clinical data (for more details see Supplementary Material).

\subsection{PET data acquisition and processing}

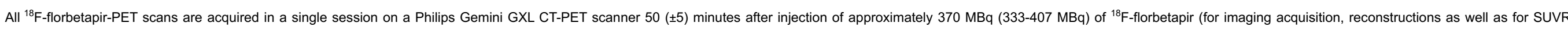




\subsection{Statistical modeling}

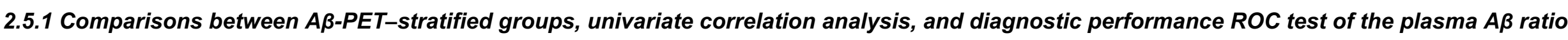

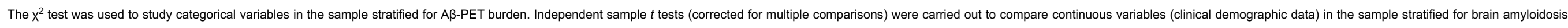
Normality of variables and residuals and heteroskedasticity were checked visually. However, the samples size wasis large enough for the $t$ test to be valid even if all variables weare not normally distributed.

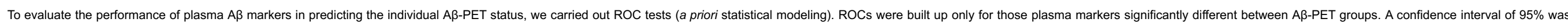

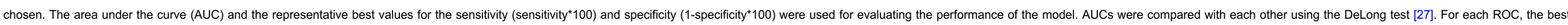

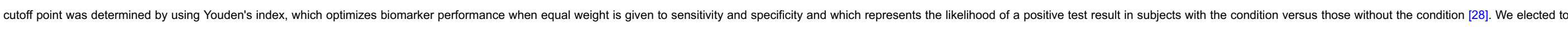

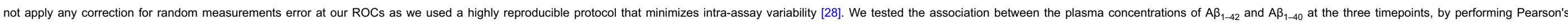

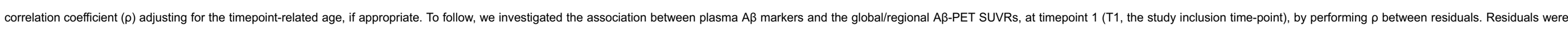
recovered through carrying out a linear regression analysis between each dependent variable (either plasma markers or regional SUVRs) and age plus sex as independent variables.

All tests were two-tailed, and $P$ values $<0.05$ were considered statistically significant. The Bonferroni correction was applied to adjust the false discovery rate for multiple comparisons. Data analysis was performed using the SPSS 23.0 IBM software for macOS

\subsubsection{Tree-based analysis of time-series data}

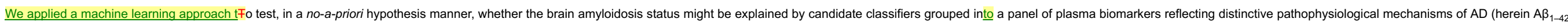

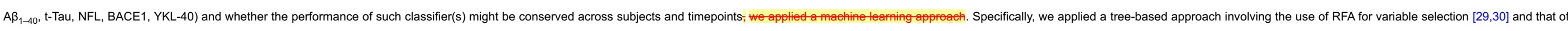

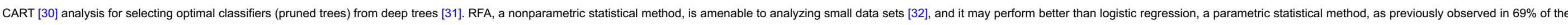

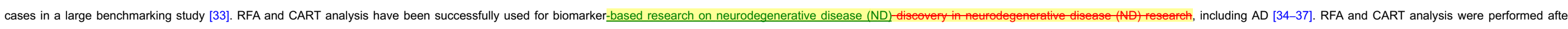

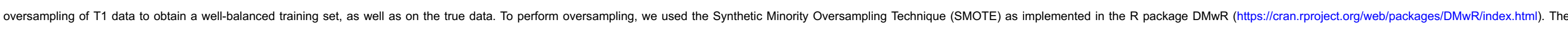

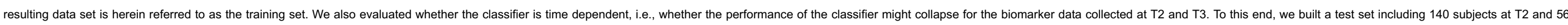
additional subjects at $\mathrm{T} 3$.

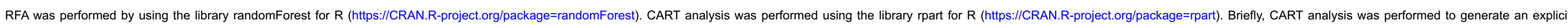

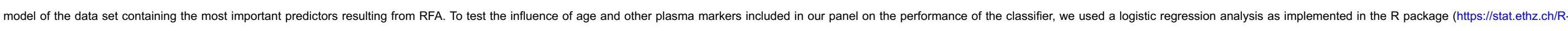
manual/R-devel/library/stats/htm//00Index.html).

\section{Results}

\subsection{Association between plasma $A \beta$ levels and brain amyloidosis: Univariate and ROC analysis}

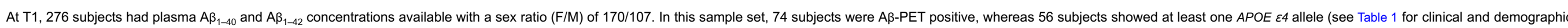

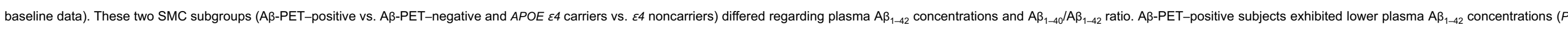

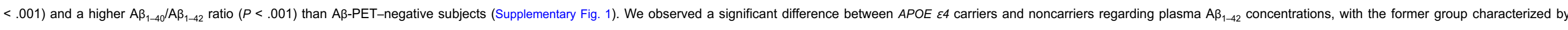

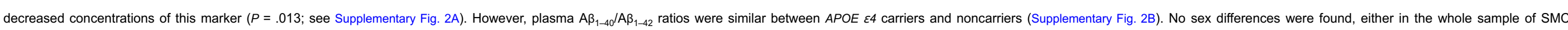

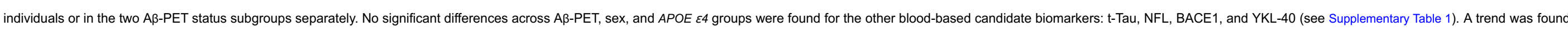

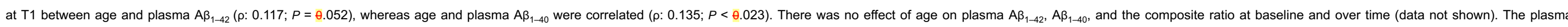
concentrations of $A \beta_{1-42}$ and $A \beta_{1-40}$ were positively correlated at each timepoint ( $\rho: 0.807 ; P<\theta .0001 \mathrm{~T} 1[\mathrm{~N}=276] ; \rho: 0.723 ; P<\theta .0001 \mathrm{~T} 2[\mathrm{~N}=215], \rho: 0.518 ; P<\theta .0001 \mathrm{~T} 3$ [N = 134]). 


\begin{tabular}{|c|c|c|c|}
\hline & Positive $A \beta-P E T$ status $(N=73)$ & Negative $A \beta$-PET status $(N=203)$ & Statistics (df), $P$ value $*$ \\
\hline $\operatorname{Sex}(F / M)$ & $47 / 27$ & $123 / 80$ & $X^{2}=0.19(1), P=.67$ \\
\hline Age (years) & 77.3; SD 3.2 & 76.6; SD 3.4 & $\mathrm{~F}=-1,5(275), P=.13$ \\
\hline$A P O E \varepsilon 4(\mathrm{n} / \mathrm{p})$ & $46 / 27$ & $176 / 27$ & $X^{2}=18.5(1), P<\theta .001$ \\
\hline $\mathrm{A} \beta_{1-42}(\mathrm{pg} / \mathrm{mL})$ & 15.1; SD 4.0 & 18.4; SD 5.8 & $F=5,2(188,4), P<\theta .001$ \\
\hline $\mathrm{A} \beta_{1-40}(\mathrm{pg} / \mathrm{mL})$ & 295.5; SD 75.4 & 301.9; SD 87.8 & $\mathrm{~F}=0,5(274), P=.58$ \\
\hline$A \beta_{1-40} / A \beta_{1-42}$ & 19.4; SD 3.3 & 16.7; SD 5.2 & $F=-4,0(274), P<0.001$ \\
\hline Mean Aß-PET SUVR & $1.07 ;$ SD 0.27 & 0.608; SD 0.05 & $F=-11,7(74,8), P<0.001$ \\
\hline
\end{tabular}

$*$ Statistical tests are presented as test value (degree of freedom), $P$ value (significant level $P<.05$, two tailed).

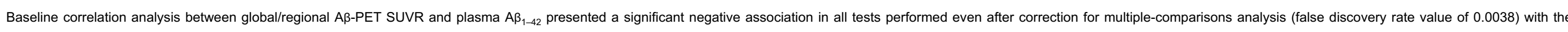

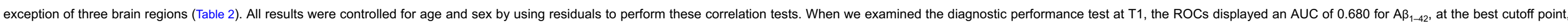

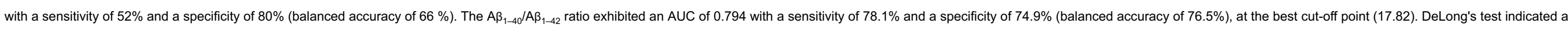
statistically significant difference between the two ROCs $(P=.006$, Fig. 1).

\section{Table 2 Correlation between regional A $\beta$-PET SUVRs and plasma A $\beta$ markers}

\begin{tabular}{|c|c|}
\hline $\begin{array}{l}\text { Regional } \\
\text { Aß-PET } \\
\text { SUVRs }\end{array}$ & $A \beta_{1-42}(p g / m L)$ \\
\hline $\begin{array}{l}\text { Cingulum } \\
\text { posterior } \mathrm{R}\end{array}$ & $\begin{array}{l}\rho=0.189 * \\
P=.0015\end{array}$ \\
\hline $\begin{array}{l}\text { Cingulum } \\
\text { posterior L }\end{array}$ & $\begin{array}{l}\rho=0.183 * \\
P=.0022\end{array}$ \\
\hline $\begin{array}{l}\text { Cingulum } \\
\text { anterior } \mathrm{R}\end{array}$ & $\begin{array}{l}\rho=0.198 * \\
P<.001\end{array}$ \\
\hline $\begin{array}{l}\text { Cingulum } \\
\text { anterior L }\end{array}$ & $\begin{array}{l}\rho=0.210 * \\
P<.001\end{array}$ \\
\hline $\begin{array}{l}\text { Frontal } \\
\text { superior } \\
\text { orbital L }\end{array}$ & $\begin{array}{l}\rho=0.183 * \\
P=.0024\end{array}$ \\
\hline $\begin{array}{l}\text { Frontal } \\
\text { superior } \\
\text { orbital R }\end{array}$ & $\begin{array}{l}\rho=0.189 * \\
P=.0015\end{array}$ \\
\hline $\begin{array}{l}\text { Parietal } \\
\text { inferior } L\end{array}$ & $\begin{array}{l}\rho=0.155 \\
P=.0099\end{array}$ \\
\hline $\begin{array}{l}\text { Parietal } \\
\text { inferior } \mathrm{R}\end{array}$ & $\begin{array}{l}\rho=0.188 * \\
P=.0016\end{array}$ \\
\hline Precuneus & $\rho=0.198 *$ \\
\hline
\end{tabular}


NOTE. Pearson's correlation coefficients seek through residuals (original value cleaned from the potential effect of age and sex).

$P$ value significant level $\alpha<0.05$, two tailed. False discovery rate: $P=\theta .0038$.

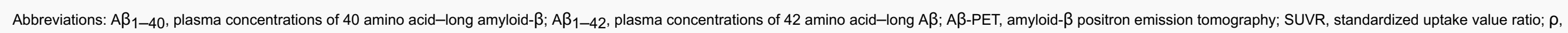
Pearson's correlation coefficient; $P$ : uncorrected $P$ value $P$ value; $R$, right; L, left.

* Significant correlations after correction for multiple comparisons.

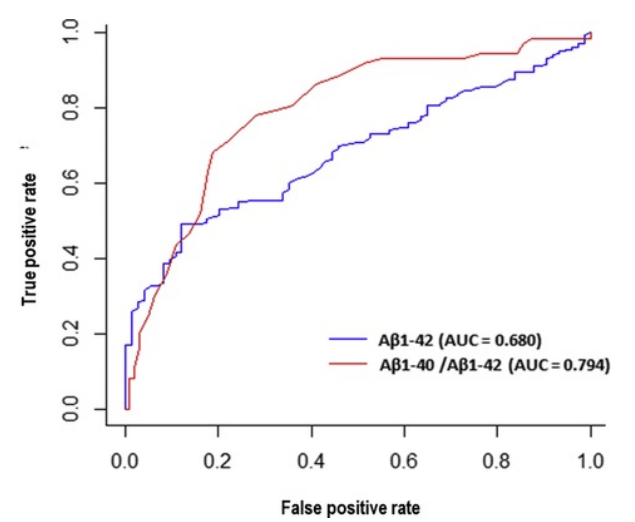

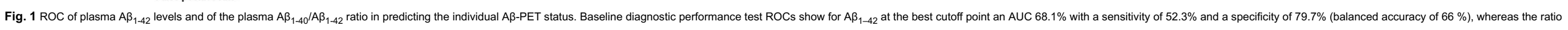

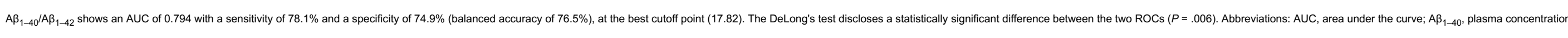
of 40 amino acid-long amyloid- $\beta ; A \beta_{1-42}$, plasma concentrations of 42 amino acid-long $A \beta ; A \beta-P E T$, amyloid- $\beta$ positron emission tomography; ROC, receiver operating characteristic curve.

\subsection{RFA and CART analysis identify plasma $A \beta_{1-40} / A \beta_{1-42}$ ratio as the best predictive variable of brain amyloidosis}

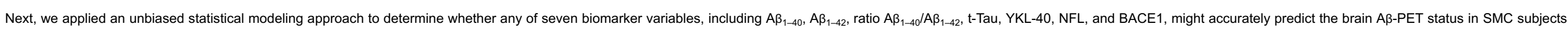

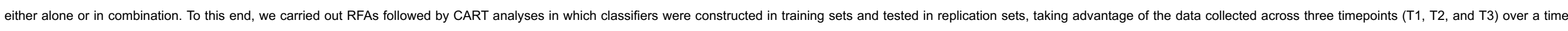
period of 3 years.

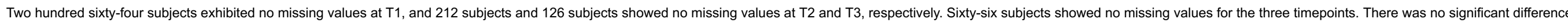
in the distribution of plasma $A \beta_{1-42}$ and ratio $A \beta_{1-40} / A \beta_{1-42}$ across timepoints.

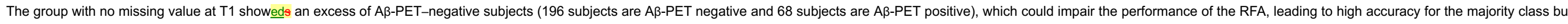

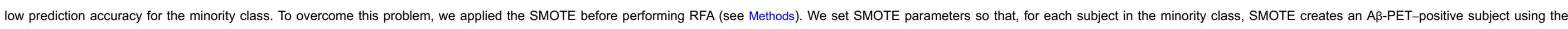

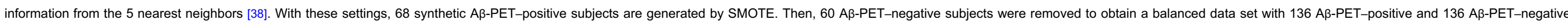




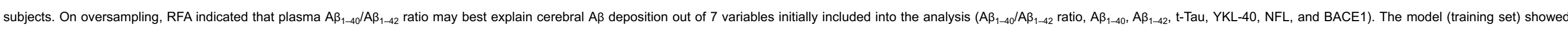

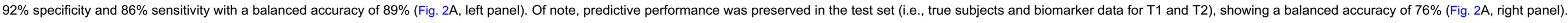
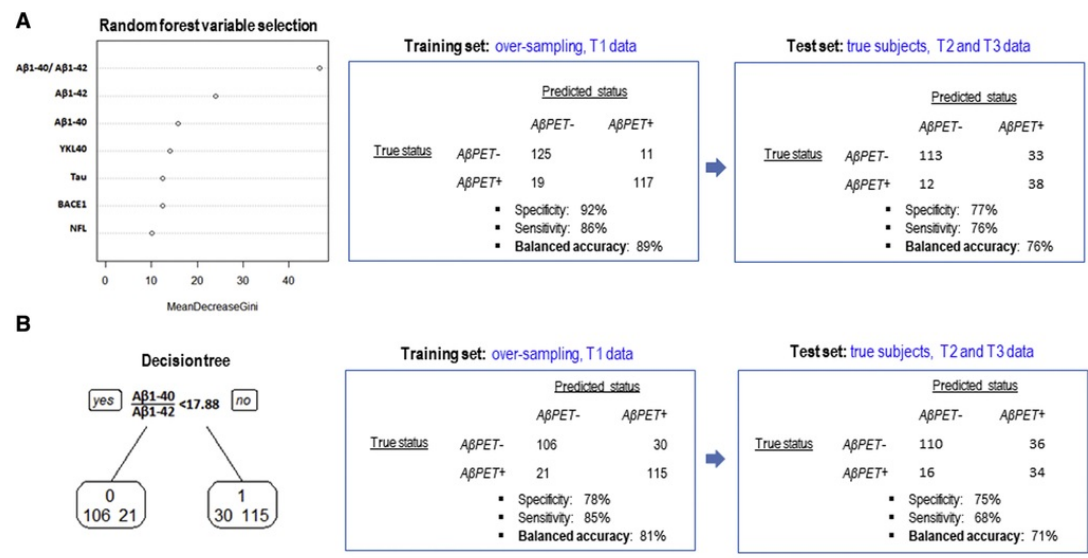

c

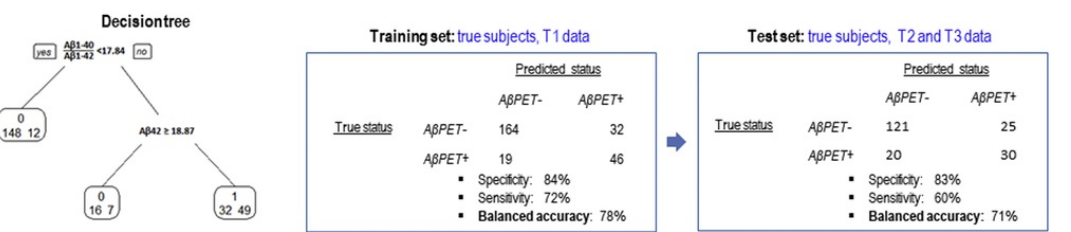

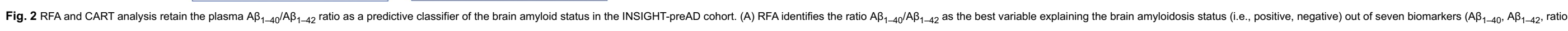

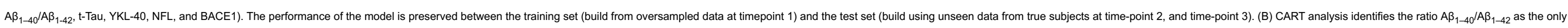

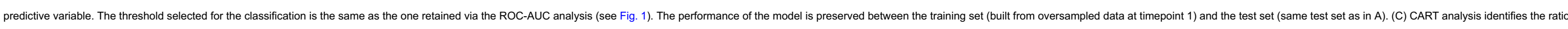

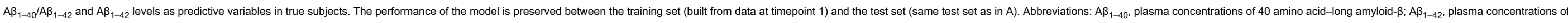

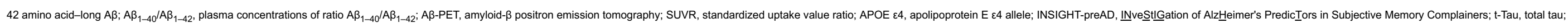
YKL-40, chitinase-like protein 1; NFL, neurofilament light chain; BACE1, $\beta$-site amyloid precursor protein cleaving enzyme 1; RFA, random forest analysis; CART, classification and regression trees.

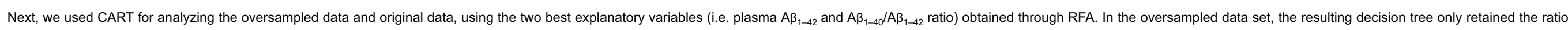

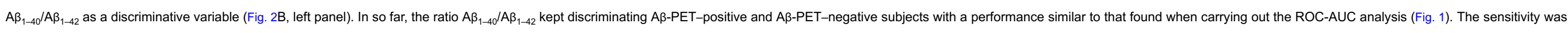

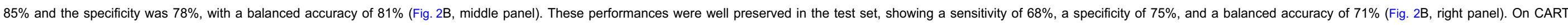

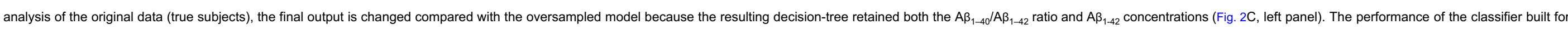

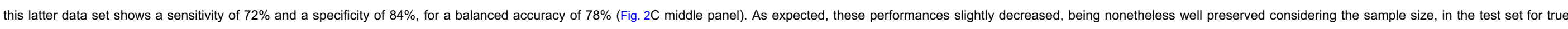
subjects because the sensitivity was $60 \%$ and specificity was $83 \%$ with a balanced accuracy of $71 \%$ (Fig. $2 \mathrm{C}$, right panel).

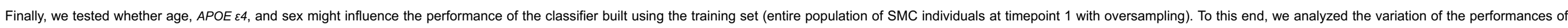

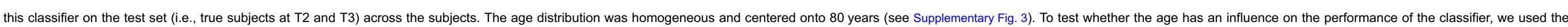

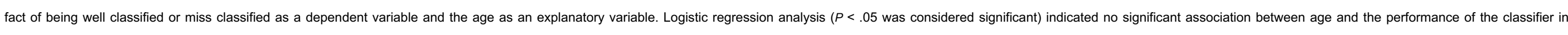
predicting brain amyloidosis $(P>.1)$, suggesting that the performance of the global CART classifier in predicting brain amyloidosis is independent of age.

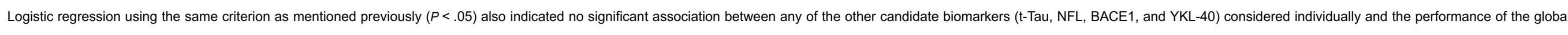

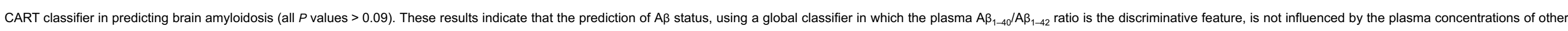




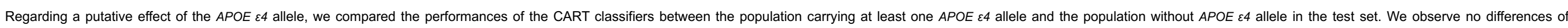

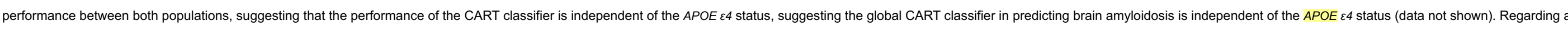

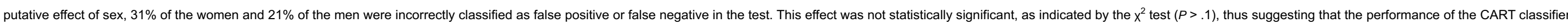
might be equally well in both populations. However, there is a trend toward females to be more often misclassified than males, which led us to further explore this question as developed below.

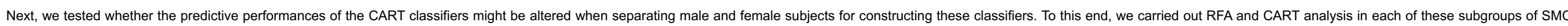
subjects, considering only the subjects with no missing value at T1 (see Supplementary Fig. 4 A-D).

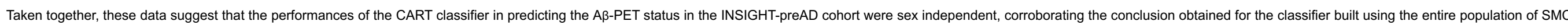
individuals.

\section{Discussion}

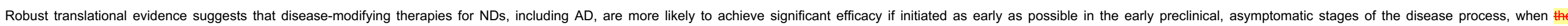

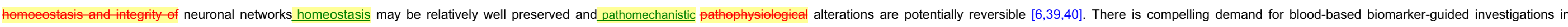

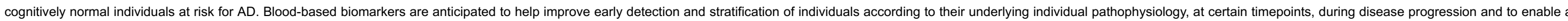

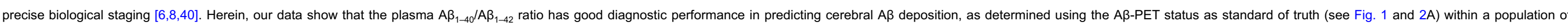

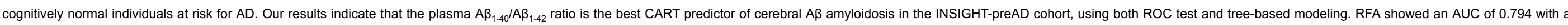

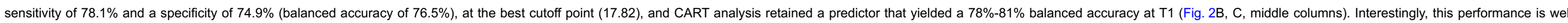

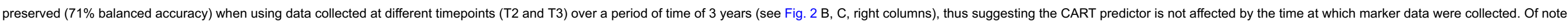
this statement should be taken carefully because no follow-up A $A-P E T$ data are available and thus the number of SMC subjects who converted to a positive A $\beta$-PET status is unknown.

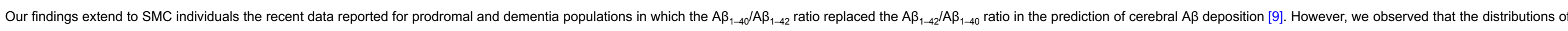

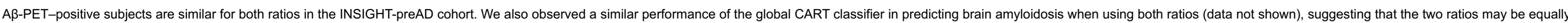
suitable for predicting brain amyloidosis

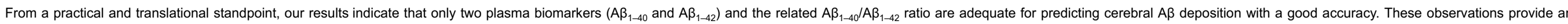

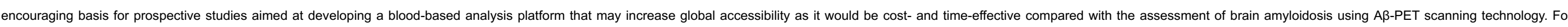

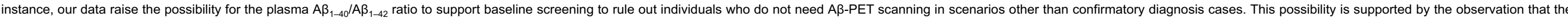
predictive performance of the plasma $A \beta_{1-40} / A \beta_{1-42}$ ratio does not appear to be affected by age, sex, and the $A P O E \varepsilon 4$ allele (although the number of $A P O E \varepsilon 4-$ positive individuals is relatively small in our study).

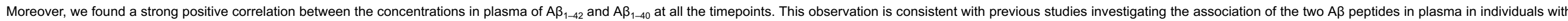
$\mathrm{MCl}$ (both converters and stables) and AD dementia [13,41].

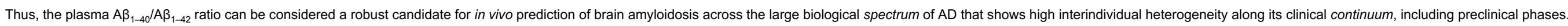

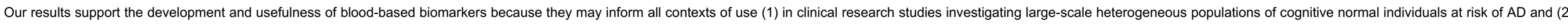

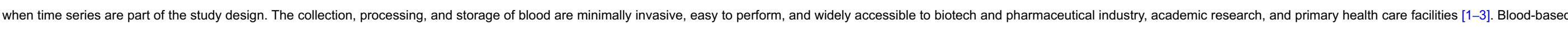

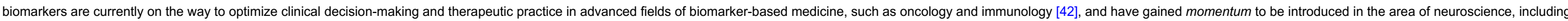
NDs such as AD.

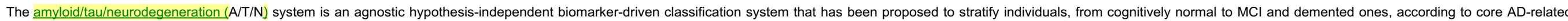
pathological and pathophysiological hallmarks [43]. The A/T/N system is based on CSF, MRI, and PET core biomarkers of AD that have been validated, qualified, and integrated into current diagnostic research criteria. 

$[3,44]$.

Moreover, although the ATN system provides key pathophysiological insights, it only partially reflects the expanding spectrum of pathomechanistic alterations occurring in AD.

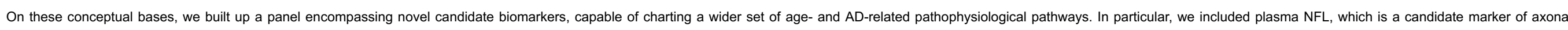

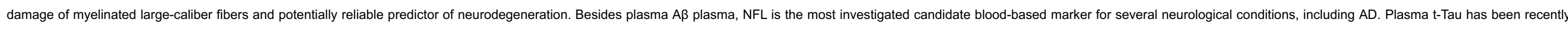

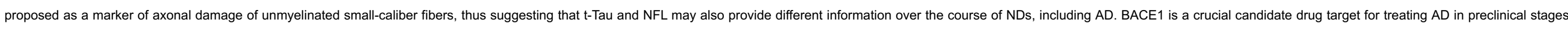

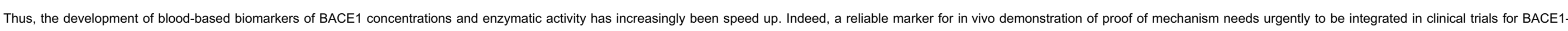

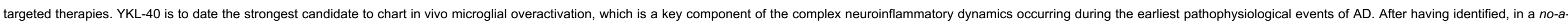

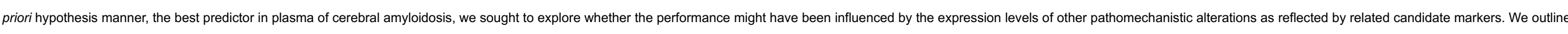

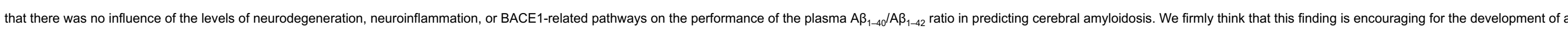

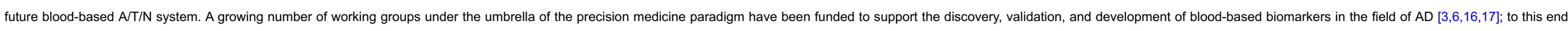

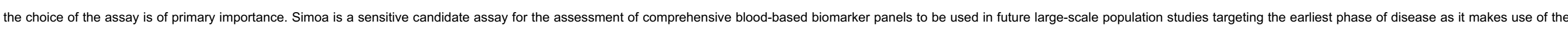

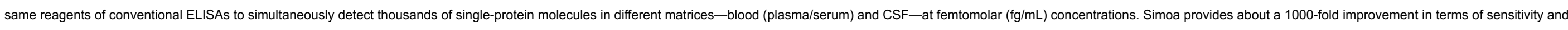
is cost-effective. As a result, a more sensitive assessment will likely be produced to get an earlier detection of the disease [45].

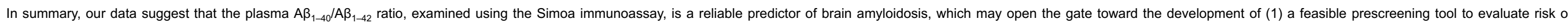
developing $A D$ in individuals with SMC in a multistage diagnostic process and (2) a valuable selection and enrichment tool for amyloid targeted clinical trials.

The relatively small size of the INSIGHT-preAD cohort, especially with regard to participants longitudinally assessed with plasma $A \beta_{1-40} / A \beta_{1-42}$ ratio measurement, represents a limit inherent to the study that deserves to be outlined.

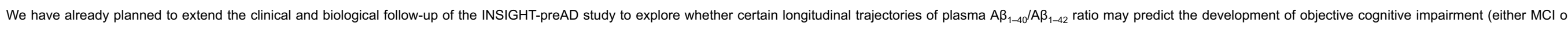

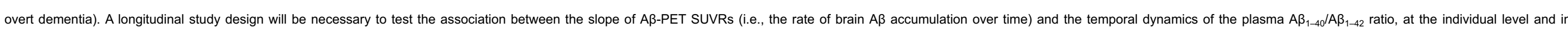

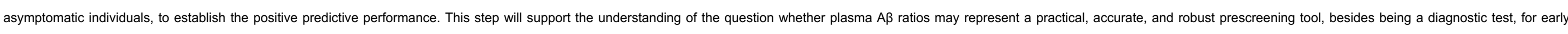

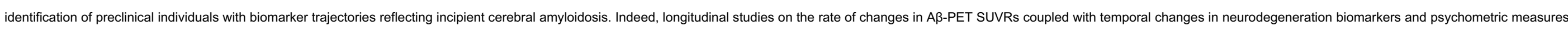

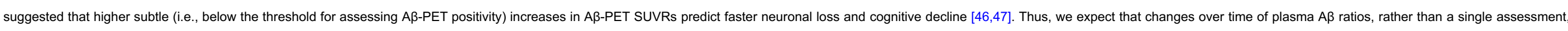

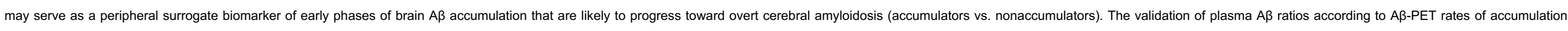
rather than the A $\beta$-PET status, represents a paradigm shift toward the investigation of anti-A $\beta$ disease-modifying compounds in large-scale populations of very early preclinical individuals, which represent the most suitable target.

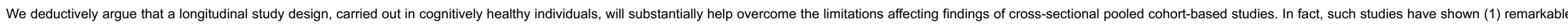

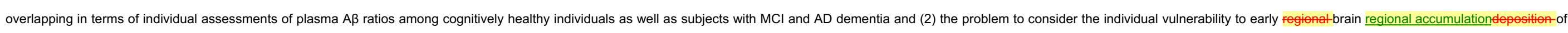
$\mathrm{A} \beta$.

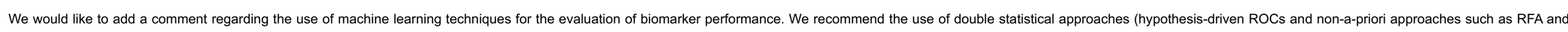

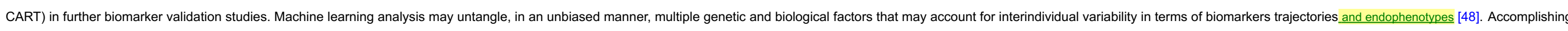
such a map of pathophysiological interactions represents a key challenge along the path to develop effective and safe drugs for precision medicine treatments [18].

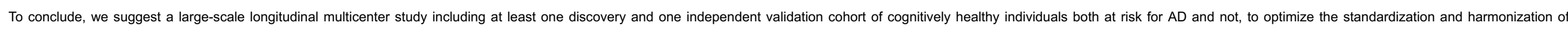

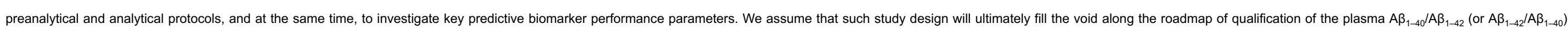
ratio as a peripheral surrogate biomarker of early cerebral amyloidosis, thus enriching both pharmacological trials and clinical management during early stages of preclinical AD. 


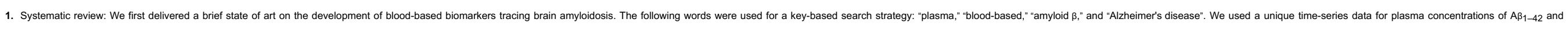

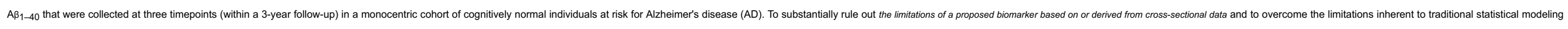

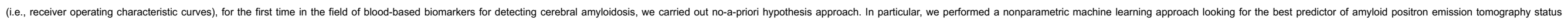

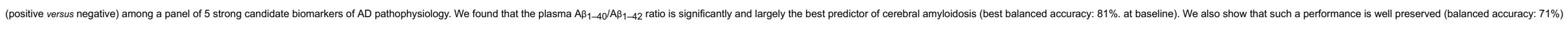
when using data collected at the subsequent time-points (after 1-year follow-up and 3-year follow-up)

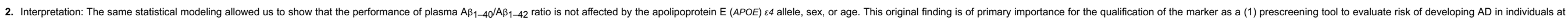
risk in a multistage diagnostic process and a (2) valuable selection and enrichment tool for amyloid targeted clinical trials.

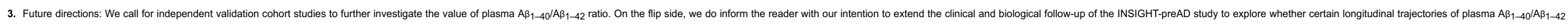
ratio may predict the development of objective cognitive impairment (either mild cognitive impairment or overt dementia).

\section{Acknowledgments}

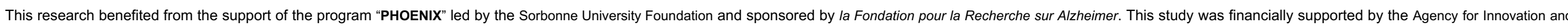

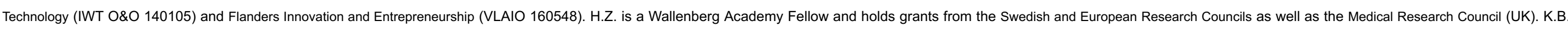

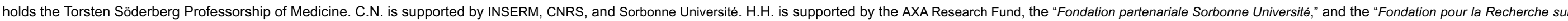

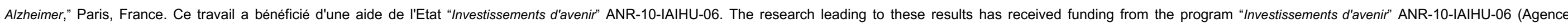
Nationale de la Recherche-10-IA Agence Institut Hospitalo-Universitaire-6).

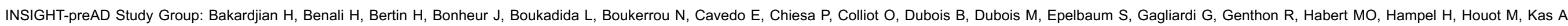
Lamari F, Levy M, Lista S, Metzinger C, Mochel F, Nyasse F, Poisson C, Potier MC, Revillon M, Santos A, Andrade KS, Sole M, Surtee M, Thiebaud de Schotten M, Vergallo A, and Younsi N.

INSIGHT-preAD Scientific Committee Members: Dubois B, Hampel H, Bakardjian H, Benali H, Colliot O, Habert Marie-O, Lamari F, Mochel F, Potier MC, and Thiebaut de Schotten M.

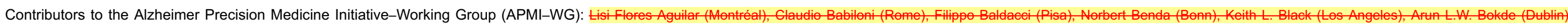

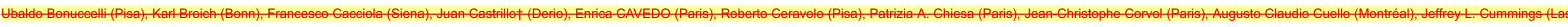

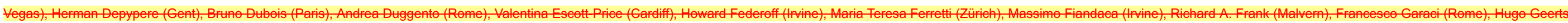

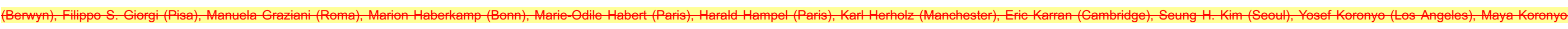

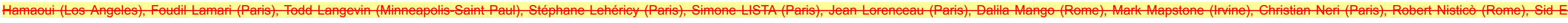

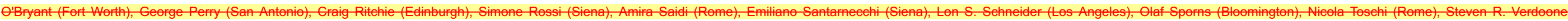

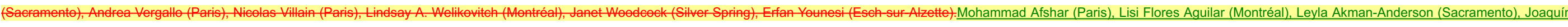

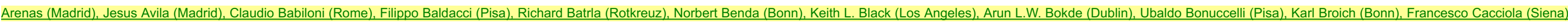

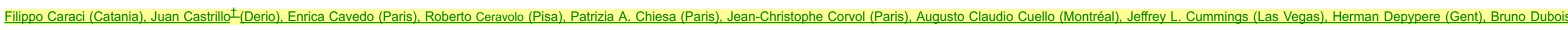

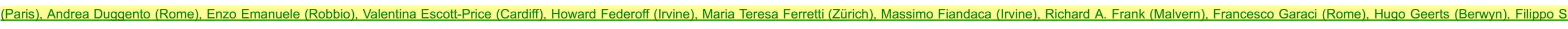

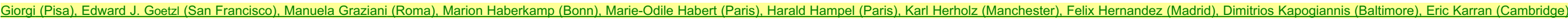

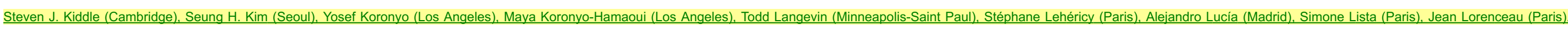

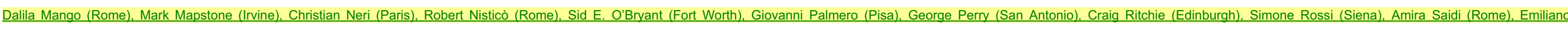

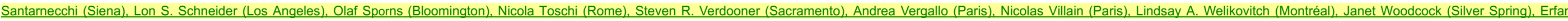
Younesi (Esch-sur-Alzette).

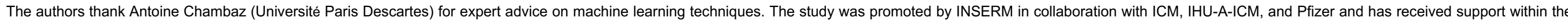

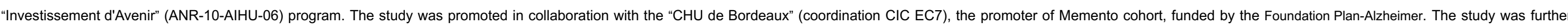




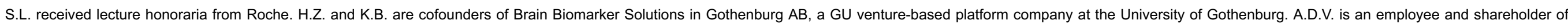

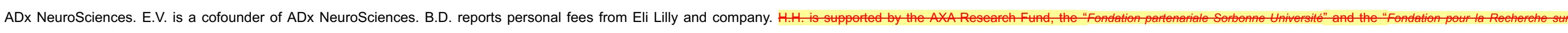

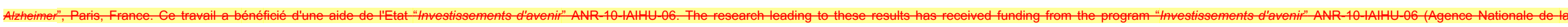

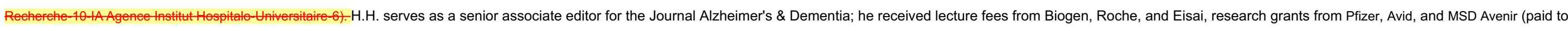

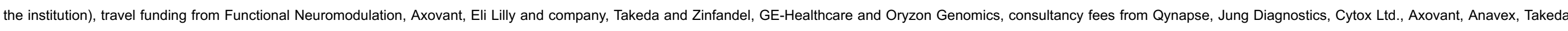

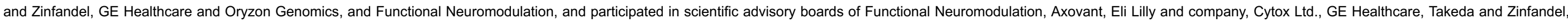

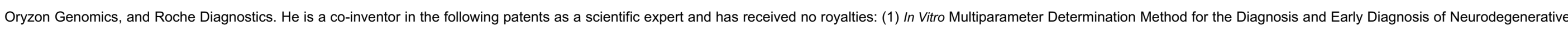

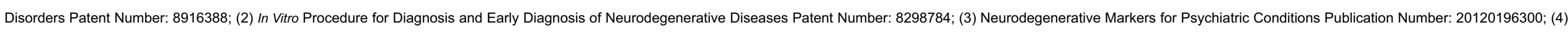

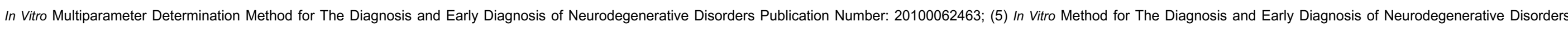

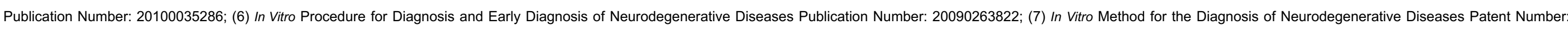

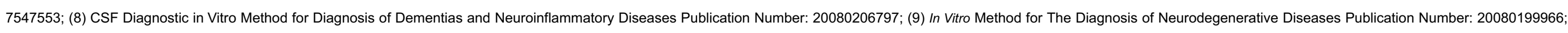
and (10) Neurodegenerative Markers for Psychiatric Conditions Publication Number: 20080131921 . A.V., L.M., C.N., M.-O.H., M.-C.P., and E.C. declared no conflicts of interest.

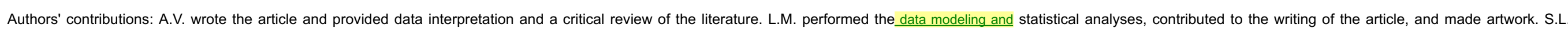

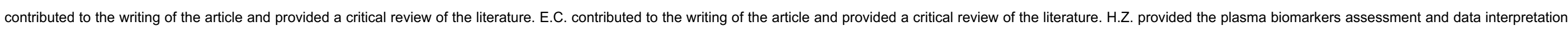

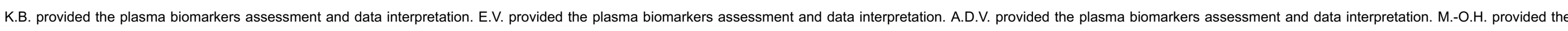

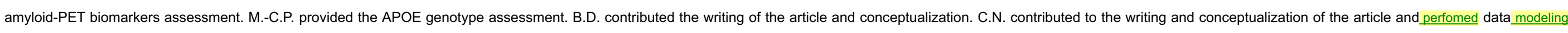
and interpretation. H.H. wrote the article and provided data interpretation and a critical review of the whole manuscript.( ${ }^{\dagger}$ Deceased.)

\section{Supplementary Data}

Supplementary data related to this article can be found at https://doi.org/10.1016/j.jalz.2019.03.009.

\section{References}

[1] J. Abbasi, Plasma Biomarkers Predict Brain Amyloid-beta Burden, JAMA 319, 2018, 972.

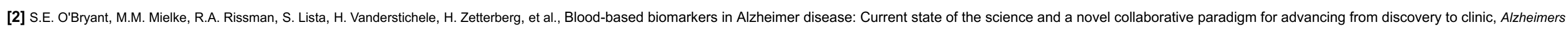
Dement 13, 2017, 45-58.

[3] H. Hampel, S.E. O'Bryant, J.L. Molinuevo, H. Zetterberg, C.L. Masters, S. Lista, et al., Blood-based biomarkers for Alzheimer disease: mapping the road to the clinic, Nat Rev Neurol 14, 2018, 639-652.

[4] J.L. Cummings, Biomarkers in Alzheimer's disease drug development, Alzheimers Dement 7, 2011, e13-e44.

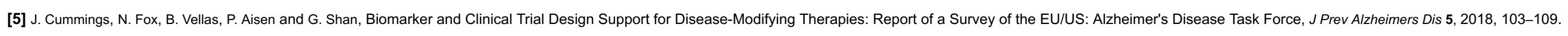

[6] H. Hampel, A. Vergallo, L.F. Aguilar, N. Benda, K. Broich, A.C. Cuello, et al., Precision pharmacology for Alzheimer's disease, Pharmacol Res 130, 2018, 331-365.

[7] H.M. Snyder, M.C. Carrillo, F. Grodstein, K. Henriksen, A. Jeromin, S. Lovestone, et al., Developing novel blood-based biomarkers for Alzheimer's disease, Alzheimers Dement 10, $2014,109-114$.

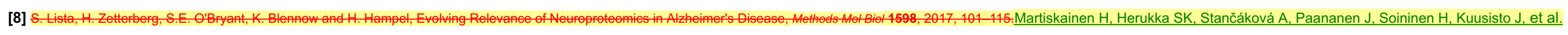
Decreased plasma $\beta$-amyloid in the Alzheimer's disease APP A673T variant carriers. Ann Neurol 2017:82:128-32.

[9] A. Nakamura, N. Kaneko, V.L. Villemagne, T. Kato, J. Doecke, V. Dore, et al., High performance plasma amyloid-beta biomarkers for Alzheimer's disease, Nature 554, $2018,249-254$. 
[10] A. Nabers, L. Perna, J. Lange, U. Mons, J. Schartner, J. Guldenhaupt, et al., Amyloid blood biomarker detects Alzheimer's disease, EMBO Mol Med 10, 2018, https://doi.org/10.15252/emmm.201708763.

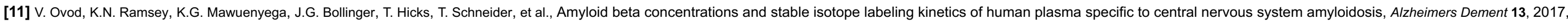
$841-849$.

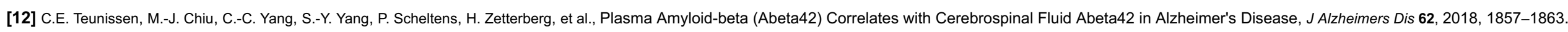

[13] O. Hanon, J.-S. Vidal, S. Lehmann, S. Bombois, B. Allinquant, J.-M. Treluyer, et al., Plasma amyloid levels within the Alzheimer's process and correlations with central biomarkers, Alzheimers Dement 14, $2018,858-868$.

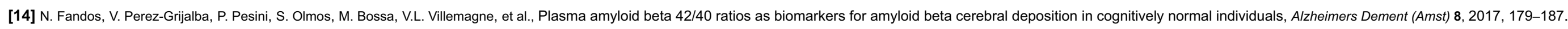

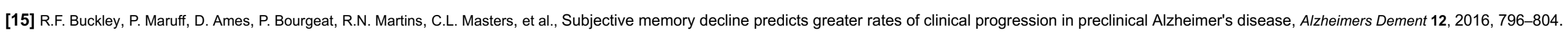

[16] A.C. van Harten, M.M. Mielke, D.M. Swenson-Dravis, C.E. Hagen, K.K. Edwards, R.O. Roberts, et al., Subjective cognitive decline and risk of MCl: The Mayo Clinic Study of Aging, Neurology 91, 2018, e300-e312.

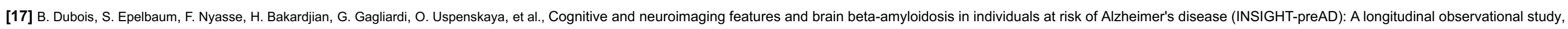
Lancet Neurol 17, 2018, 335-346.

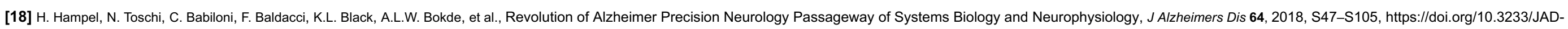
179932.

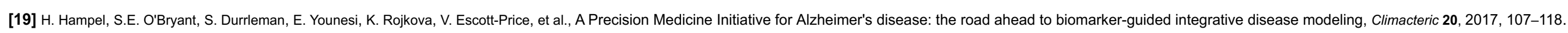

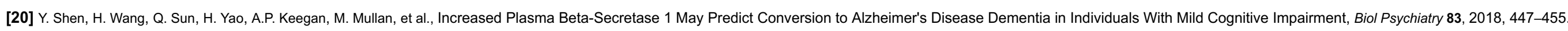

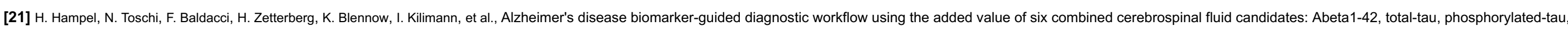
NFL, neurogranin, and YKL-40, Alzheimers Dement 14, 2018, 492-501.

[22] B. Olsson, R. Lautner, U. Andreasson, A. Ohrfelt, E. Portelius, M. Bjerke, et al., CSF and blood biomarkers for the diagnosis of Alzheimer's disease: a systematic review and meta-analysis, Lancet Neurol 15, 2016, 673-684.

[23] A. Jeppsson, M. Holtta, H. Zetterberg, K. Blennow, C. Wikkelso and M. Tullberg, Amyloid mis-metabolism in idiopathic normal pressure hydrocephalus, Fluids Barriers CNS 13, $2016,13$.

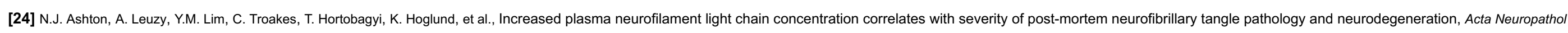
Commun 7, 2019, 5 .

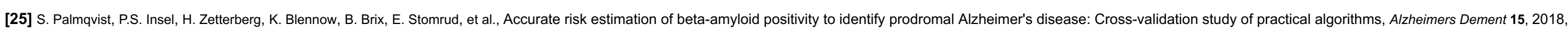
194-204.

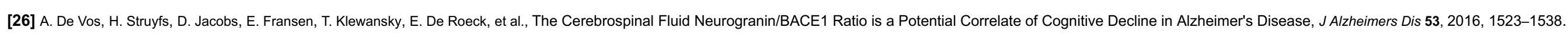

[27] E.R. DeLong, D.M. DeLong and D.L. Clarke-Pearson, Comparing the areas under two or more correlated receiver operating characteristic curves: a nonparametric approach, Biometrics 44, 1988, 837-845.

[28] N.J. Perkins and E.F. Schisterman, The Youden Index and the optimal cut-point corrected for measurement error, Biom J 47, 2005, 428-441.

[29] L. Breiman, Random Forests, Mach Learn 45, 2001, 5-32.

[30] M.J. van der Laan and S. Rose, Targeted Learning in Data Science, 2018, Springer; Cham.

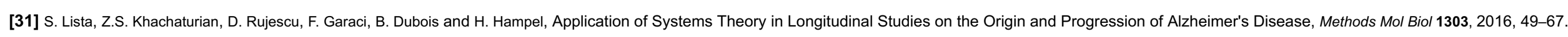

[32] T. Shaikhina, D. Lowe, S. Daga, D. Briggs, R. Higgins and N. Khovanova, Decision tree and random forest models for outcome prediction in antibody incompatible kidney transplantation, Biomed Signal Process Control 2017 https://doi.org/10.1016/j.bspc.2017.01.012. 
[33] R. Couronne, P. Probst and A.-L. Boulesteix, Random forest versus logistic regression: a large-scale benchmark experiment, BMC Bioinformatics 19, $2018,270$.

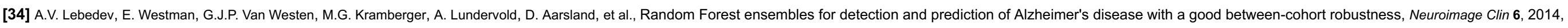
$115-125$.

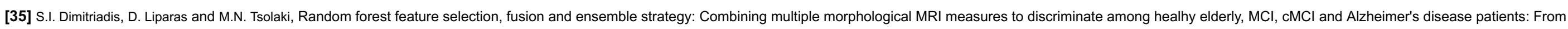
the Alzheimer's disease neuroimaging initiative (ADNI) data, J Neurosci Methods 302, 2018, 14-23.

[36] H. Kaduszkiewicz, M. Eisele, B. Wiese, J. Prokein, M. Luppa, T. Luck, et al., Prognosis of mild cognitive impairment in general practice: results of the German AgeCoDe study, Ann Fam Med 12, 2014, 158-165.

[37] A. Besga, I. Gonzalez, E. Echeburua, A. Savio, B. Ayerdi, D. Chyzhyk, et al., Discrimination between Alzheimer's Disease and Late Onset Bipolar Disorder Using Multivariate Analysis, Front Aging Neurosci 7, $2015,231$.

[38] N.V. Chawla, K.W. Bowyer, L.O. Hall and W.P. Kegelmeyer, SMOTE: Synthetic Minority Over-sampling Technique, J Artif Int Res 16, 2002, 321-357.

[39] P.S. Aisen, B. Vellas and H. Hampel, Moving towards early clinical trials for amyloid-targeted therapy in Alzheimer's disease, Nat Rev Drug Discov 12, $2013,324$.

[40] R.A. Sperling, J. Karlawish and K.A. Johnson, Preclinical Alzheimer disease-the challenges ahead, Nat Rev Neurol 9, 2013, 54-58.

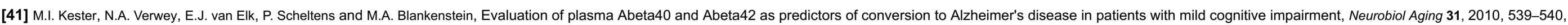
author reply 541.

[42] V.B. Kraus, Biomarkers as drug development tools: discovery, validation, qualification and use, Nat Rev Rheumatol 14, 2018, 354-362.

[43] C.R.J. Jack, D.A. Bennett, K. Blennow, M.C. Carrillo, B. Dunn, S.B. Haeberlein, et al., NIA-AA Research Framework: Toward a biological definition of Alzheimer's disease, Alzheimers Dement 14, 2018 , 535-562.

[44] J.L. Molinuevo, S. Ayton, R. Batrla, M.M. Bednar, T. Bittner, J. Cummings, et al., Current state of Alzheimer's fluid biomarkers, Acta Neuropathol 136, 2018, 821-853.

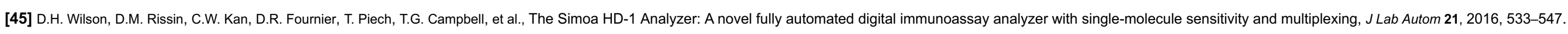

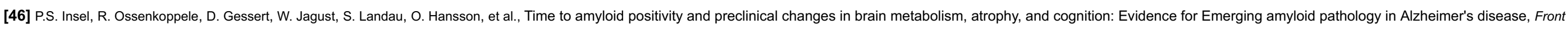
Neurosci 11, 2017, 281.

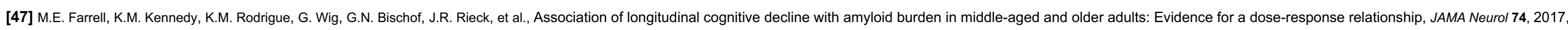
830-838.

[48] B. Goudey, B.J. Fung, C. Schieber and N.G. Faux, A blood-based signature of cerebrospinal fluid Abeta1-42 status, Sci Rep 9, $2019,4163$.

\section{Supplementary data}

Multimedia Component 1

Supplementary Materaial

\section{Queries and Answers}

Query: If there are any drug dosages in your article, please verify them and indicate that you have done so by initialing this query Answer: No drug dosages reported within the manuscript text

Query: Please provide fax number for the corresponding author.

Answer: Fax number added: +3315727 4027 
Query: Please provide address details and affiliations for all authors listed.

Answer: Affiliations edited as follows (see also attached file):

Andrea Vergallo $o^{a, b, c^{*}}$, Lucile Mégret ${ }^{d}$, Simone Lista ${ }^{a, b, c}$, Enrica Cavedo a,b,c,

Henrik Zetterberg ${ }^{e, f, g, h}$, Kaj Blennow ${ }^{e, f}$, Eugeen Vanmecheleni, Ann De Vosi,

Marie-Odile Habert ${ }^{\mathrm{j}, \mathrm{k}, \mathrm{l}}$, Marie-Claude Potier ${ }^{\mathrm{m}}$, Bruno Dubois ${ }^{\mathrm{a}, \mathrm{b}, \mathrm{c}}$,

Christian Nerid, and Harald Hampel $\mathrm{l}^{\mathrm{a}^{*}}$

and the INSIGHT-preAD study group

for the Alzheimer Precision Medicine Initiative (APMI)

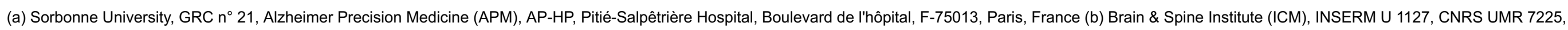

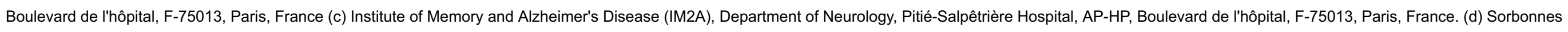

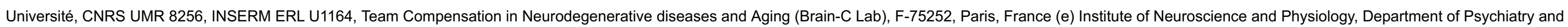

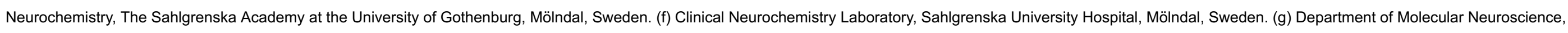

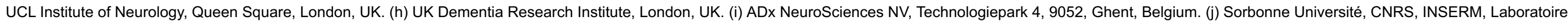

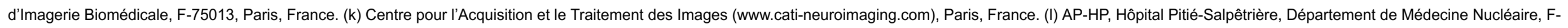
75013, Paris, France. (m) ICM Institut du Cerveau et de la Moelle épinière, CNRS UMR7225, INSERM U1127, UPMC, Hôpital de la Pitié-Salpêtrière, 47 Bd de l'Hôpital, F-75013 Paris, France

Attachments: affiliations_edited.docx

Query: Please check the hierarchy of section heads for this article.

Answer: We confirm the hierarchy is fine

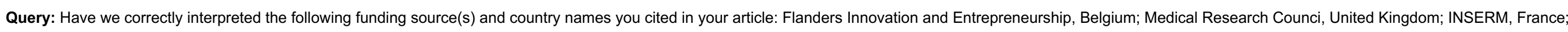
CNRS, France; Sorbonne Université, France; AXA Research Fund, France; Pfizer, United States; Lilly, United states?

Answer: Yes

Query: Please mention the section head instead of the term "below" given in the sentence "Time series data for plasma...."

Answer: Text edited accordingly

Query: Please check the edits made to the sentence "In addition, four novel candidate surrogate..." and amend if necessary.

Answer: The sentence is fine

Query: Please check the edits made to the sentence "Exclusion criterion was having..." and amend if necessary.

Answer: The sentence is fine

Query: Please check the edits made to the sentence "The plasma concentrations of $A \beta_{1-42} \ldots$ " and amend if necessary.

Answer: The sentence is fine

Query: Please check the edits made to the sentence "Of note, this statement should be..." and amend if necessary.

Answer: The statement is fine 
Query: Please provide the expansion of "A/T/N system" given in the sentence "The A/T/N system is an agnostic...."

Answer: Please find below a suggested expansion: The "A (amyloid) / T (tau) / N (neurodegeneration)" system

Query: Please check the edits made to the sentence "After having identified, in a no-a-priori hypothesis..." and amend if necessary.

Answer: The sentence is fine

Query: Please check the edits made in "Acknowledgments" and "Conflicts of interest" and amend if necessary.

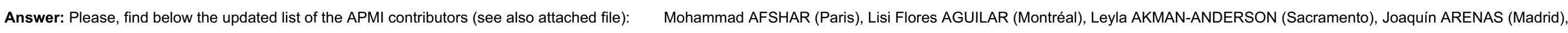

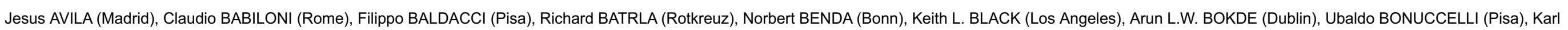

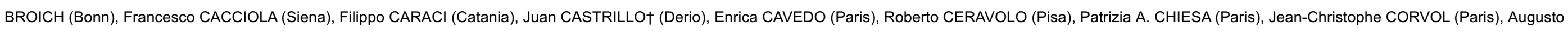

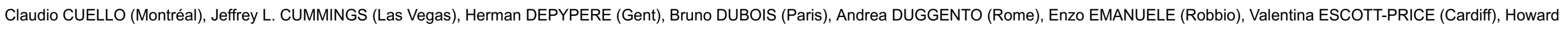

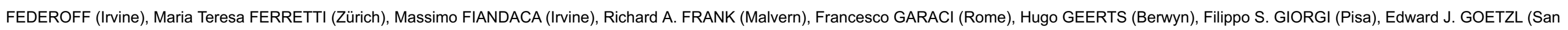

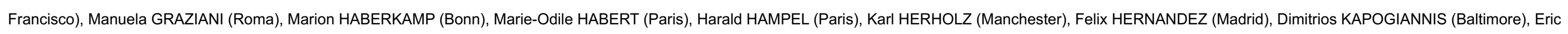

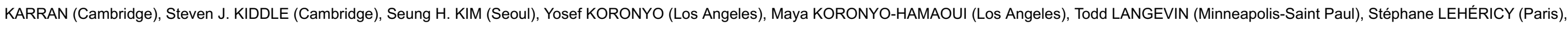

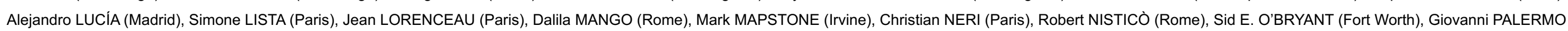

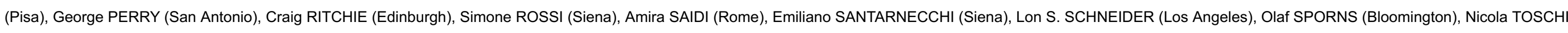

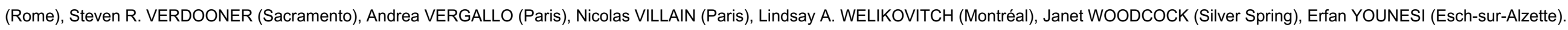

Attachments: Contributors to the Alzheimer Precision Medicine I

Query: Please check the edits made in "Tables 1-2" and amend if necessary.

Answer: Tables 2 and 2 are fine

Query: Please provide a definition for the significance of dagger in the acknowledgement.

Answer: $†$ stands for "passed away"

Query: Please verify the in-text citation of Refs. 7-14, amend if necessary.

Answer: Reference 8 is incorrect and needs to be replaced. Correct reference: Martiskainen H et al. Decreased plasma $\beta$-amyloid in the Alzheimer's disease APP A673T variant carriers. Ann Neurol. 2017

Query: Please provide city for affiliation 'l'.

Answer: Paris. Text edited

Query: Please confirm that given names and surnames have been identified correctly and are presented in the desired order and please carefully verify the spelling of all authors' names.

Answer: Yes 